\title{
Importance Des Produits Forestiers Non Ligneux Medicinaux D'origine Vegetale Et Impacts Des Activites Anthropiques Sur Leur Durabilite Dans Le Sud-Ouest De La Republique Centrafricaine
}

\author{
Guy Gildas Zima, \\ Université de Bangui, Faculté des Sciences, \\ Département de la Biodiversité Végétale, République Centrafricaine \\ Fidèle Mialoundama, \\ Université Marien Ngouabi, Faculté des Sciences-Formation Doctorale \\ Sciences Naturelles Agronomie, Brazzaville, Congo \\ Jean Michel Yangakola, \\ Université de Bangui, Faculté des Sciences, \\ Département de la Biodiversité Végétale, République Centrafricaine \\ Innocent Kossa, \\ Institut Supérieur de Développement Rural (ISDR), \\ Université de Bangui, Mbaiki, Centrafrique
}

Doi: 10.19044/esj.2018.v14n33p202 URL:http://dx.doi.org/10.19044/esj.2018.v14n33p202

\begin{abstract}
The forest in the south-west of the Central African Republic has enormous potential for non-timber forest products (NTFPs) of plant origin. The objective of this study is to identify the medicinal value of these NTFPs of plant origin with high health potential and the effects of human activities on their sustainability. The study was carried out in 09 villages in the intervention zone of the Kadéi Forestry Company. From various methodological approaches (ethno botanical survey, participant observation, inventory technique), data were collected, processed and analysed. From the results obtained 91 non-wood medicinal plant species belonging to 29 families and to one large systematic group, the Magnoliophytes were identified. These species represent a means of therapeutic remedies used by $91 \%$ of surveyed populations to treat many diseases, symptoms and infections. Tree barks (43.26\%) and leaves (39.21\%) are the most commonly harvested organs. The variables collected related to the types of uses allowed to identify 19 priority NTFPs medicinal and underline the process of degradation of 7 important NTFPs. This degradation is mainly due to the way in which the natural resource is harvested, causing a large number of non-
\end{abstract}


woody medicinal plant species to become rare. The definition of strategies based on ecological and socioeconomic harvesting techniques of NTFPs are solutions to ensure sustainable management of these products for the benefit of future generations.

Keywords: NTFPs, uses, human activities, sustainability, Central African Republic

\section{Resume}

La forêt du Sud-Ouest de la République Centrafricaine regorge d'énormes potentialités en produits forestiers non ligneux (PFNL) d'origine végétale. L'objectif de cette étude est d'identifier les PFNL médicinaux d'origine végétale à fort potentiel sanitaire et les effets des activités anthropiques sur leur durabilité. L'étude a été réalisée dans 09 villages de la zone d'intervention de la Société Forestière de Kadéi. À partir de diverses approches méthodologiques (enquête ethnobotanique, revue bibliographique, technique d'inventaires), des données ont été collectées, traitées et analysées. Les résultats obtenus ont permis de recenser 91 espèces végétales non ligneuses médicinales appartenant à 29 familles et à 1 grand groupe systématique à savoir les Magnoliophytes. Ces espèces représentent un moyen de recours thérapeutique utilisé par $91 \%$ de populations enquêtées pour soigner de nombreux maladies, symptômes et affections. Les écorces $(43,26 \%)$ et les feuilles $(39,21 \%)$ sont les organes les plus prélevés par les populations. Les variables recueillies à partir des types d'usages ont permis d'identifier 19 PFNL prioritaires et souligner la dégradation de 7 importants PFNL. Cette dégradation est due surtout à l'exploitation abusive et au mode de prélèvement causant ainsi la raréfaction des PFNL médicinaux. La définition des stratégies basée sur les techniques de prélèvement écologique et socioéconomique des PFNL constitue des solutions pour assurer une gestion durable de ces produits au profit des générations futures.

Mots clés : PFNL, utilisations, activités anthropiques, durabilité, République Centrafricaine

\section{Introduction}

Les forêts d'Afrique Centrale couvrent une superficie d'environ 241 millions d'hectares (FAO, 2003), dont plus de la moitié représente la forêt du bassin du Congo qui constitue la deuxième plus grande couverture forestière dense humide du monde après l'Amazonie, soit $12 \%$ du couvert forestier tropical. Les forêts constituent des écosystèmes particulièrement précieux pour l'humanité. Elles représentent un réservoir de biodiversité végétale et 
animale dont les populations rurales et urbaines profitent pour leur alimentation, leur habitat et leur santé (Drabo-Ibrahima, 2006).

Le massif forestier du Sud-Ouest de la République Centrafricaine (RCA) occupe une superficie de 3,8 millions d'hectares (ha) soit environ $15 \%$ de la superficie du territoire national. Cet écosystème possède une flore médicinale riche et contribue au moyen d'existence des populations rurales, généralement pauvres, qui vivent à l'intérieur ou à sa périphérie. Du fait de manque des infrastructures sanitaires dans la majorité des villages du SudOuest de la RCA, le nombre de populations utilisant actuellement les plantes médicinales est très important et croît de plus en plus depuis la crise militaropolitique qui a secoué le pays depuis deux décennies. Selon l'Organisation mondiale de la santé (OMS), $80 \%$ des populations rurales et même urbaines d'Afrique noire font recours aux plantes médicinales pour leurs soins de santé (Abayomi, 1996). Les plantes médicinales sont utilisées par les populations pour soigner de nombreuses maladies. Certaines d'entre elles ne sont pas exclusivement utilisées pour les soins médicaux humains, mais également appliquées en médecine vétérinaire, comme plantes toxiques utilisées comme pesticide, poison de flèche ou de pêche, ou encore comme narcotique (PROTA, 2008).

L'utilisation durable des plantes médicinales ne pourra bien se faire que si elle est bien connue des populations riveraines, d'où la nécessité de mener des études sur la flore, notamment celle dite médicinale. Brenan (1963) signale que la flore médicinale est indispensable pour les pays en voie de développement que pour les pays développés. Il est urgent de mener des recherches par l'approche participative, pour combler les lacunes dans nos connaissances ethnobotaniques et ethnoécologiques sur les PFNL à usage médicinal abritant le massif forestier du sud-ouest de la RCA. Le problème principal dans ce massif est l'exploitation non rationnelle des PFNL à usage médicinal. La problématique de notre étude réside sur un seul point : quel est l'impact réel des activités anthropiques sur la disponibilité en produits forestiers non ligneux d'origine végétale à usage médicinal dans le Sud-Ouest de la République Centrafricaine? Les résultats de ce travail pourront nous permettre de répondre à cette principale question.

L'objectif de l'étude est d'identifier les PFNL d'origine végétale à fort potentiel médicinal (sanitaire) et mesurer l'impact des activités anthropiques sur leur durabilité. L'hypothèse de cette étude est basée sur : «les activités anthropiques contribuent à la gestion durable des PFNL médicinal d'origine végétale dans le Sud-Ouest de la RCA ». 


\section{Méthodologie}

Zone d'étude

L'étude a été réalisée dans la concession de la société forestière de kadéi (SFK). Elle exploite le Permis d'Exploitation et d'Aménagement (PEA) 175 qui se situe géographiquement entre $3^{\circ} 40^{\prime}$ et $4^{\circ} 04^{\prime}$ de latitude Nord et $15^{\circ} 04^{\prime}$ et $15^{\circ} 32^{\prime}$ de longitude Est. Le PEA 175 se localise dans la partie sudouest de la préfecture de la Mambéré Kadei plus précisément dans les souspréfectures de Sosso Nakombo et Dédé Mokouba. Cette préfecture s'étend sur environ $30150 \mathrm{~km}^{2}$ avec une population totale estimée à 764795 habitants (RGPH, 2010), soit une densité moyenne de 12,1 habitants au $\mathrm{km}^{2}$ et compte environ 75 villages.

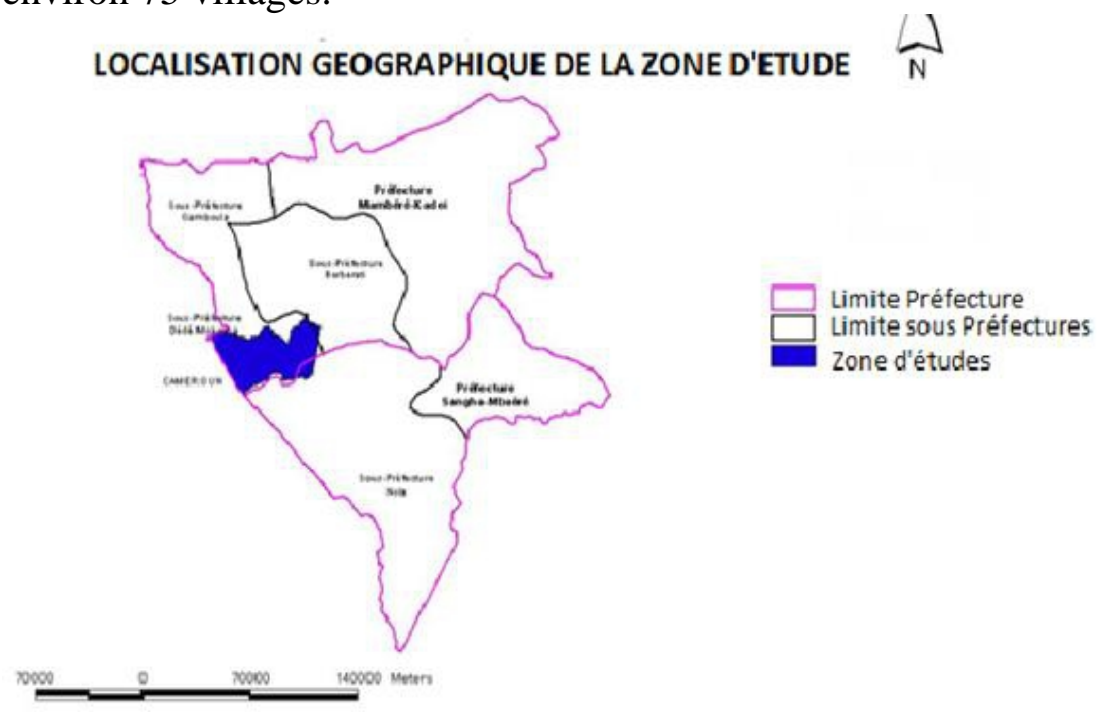

Figure 1 : localisation géographique de la zone d'études

\section{Collecte des données}

La méthodologie comprend deux grands volets. Le premier concerne la revue de la littérature. Elle a permis non seulement de cerner la manière dont les précédents travaux ont abordé la question de l'importance et impact des activités anthropiques sur la durabilité des produits forestiers non ligneux médicinaux d'origine végétale en République Centrafricaine, ainsi qu'ailleurs, mais aussi d'en relever les insuffisances. Le deuxième volet a consisté en la collecte des données de terrain qui s'est déroulée pendant la période allant du Septembre 2009 à Février 2010 et à leur traitement. Un questionnaire conçu en fonction des objectifs de l'étude a alors été administré (entretien semi directif) permettant de collecter les données sociales, culturelles et économiques. Le logiciel Microsoft Excel a été utilisé pour dépouiller le questionnaire et pour analyser les résultats d'une part; tandis que l'Indice de 
Pertinence Culturelle (IPC) a été aussi utilisé pour permettre de déterminer de façon significative les espèces ayant une grande valeur d'utilisation. Ces étapes ont permis d'apprécier les différents modes de prélèvements et d'usages des PFNL médicinaux, ainsi que les impacts écologiques sur ces ressources biologiques.

Les populations qui vivent dans ou proche de ses neuf (9) villages exploitent de façon abusive les PFNL médicinaux poussant dans les sept (7) types d'utilisation de terre (forêt secondaire âgée, forêt secondaire jeune, jachère âgée, jachère jeune, plantation caféière, marécage) identifiée suivant un dispositif d'inventaire constitué de 14 parcelles d'une superficie de $2500 \mathrm{~m}^{2}(50 \mathrm{~m} \times 50 \mathrm{~m})$. L'exploitation intense de ces PFNL se fait à l'aide d'outils rudimentaires. Elle concerne surtout les feuilles, les écorces et les racines.

\section{Résultats}

\section{Caractéristiques socio-économiques des exploitants des PFNL médicinaux}

Elles ont été faites à base des données recueillies dans neuf (09) villages sur 75 que compte le PEA 175 de la SFK. Il s'agit de Bamba, Djambala, M'bi, Binoumbi, Ngola, Djilo mégombang, Yandoa, Gnemélé et à Bayanga ngombé. Ces villages représentent la zone à plus forte densité de production des PFNL où vit la plus grande partie des populations Bantous et autochtones (29 486 personnes : Mairie de Bamaba ; 2012). Dans ces villages, l'âge des ménages enquêtés varie entre 20 et 80 ans. La moyenne d'âge sur l'ensemble de l'échantillon est de 43 ans. La tranche d'âge de 40 à 60 ans est majoritaire et constitue environ $44 \%$ du total de 516 ménages enquêtés. Il s'agit d'adultes en âge de procréer, faisant également partie de la couche paysanne active du terroir. Suivi des jeunes dans la tranche d'âge varie de 20 à 40 ans et représentent $37 \%$ du total d'échantillons enquêtés, qui sont à la quête de savoir sur l'utilisation, la valorisation et la gestion des PFNL dans leur localité. Par contre, la classe d'âge [60-80[ans est minoritaire et représente $19 \%$ des personnes enquêtées. Il s'agit des vieillards dont le savoir et la connaissance en matière des espèces forestières non ligneuses constituent un atout indispensable pour une meilleure préservation de la ressource naturelle. Ces mêmes résultats ont permis de déterminer le niveau d'instruction des ménages vivant dans le neuf (09) villages. On dénombre 79,36\% de ménages non instruits, contre $20,64 \%$ de ménages dont le niveau d'instruction correspond au moins à l'obtention d'un diplôme (11\% CEPE; 7\% BEPC et $3 \% \mathrm{BAC})$. La répartition des enquêtés selon le concept genre montre que les hommes $(67,27 \%)$ sont plus représentés que les femmes $(32,73 \%)$. Dans le sud-ouest de la RCA et plus précisément dans la zone de notre étude, l'homme en tant que chef de la famille a le droit de recevoir des visiteurs dans la maison, 
surtout pour des questions d'intérêt communautaire visant le développement local. Les origines ethniques des exploitants des PFNL présentent une grande hétérogénéité même si cinq (5) groupes sont fortement représentés. Il s'agit des Gbaya, Mbémou, Gbaya Yanguéré, Peulh et Bayaka.

\section{Inventaires floristiques des produits forestiers non ligneux à usage médicinal}

Les plantes médicinales contribuent de façon significative à la vie des populations rurales et à leurs équilibres socioéconomiques. ÀA partir des dispositifs de collecte des données mis en place, 91 espèces végétales non ligneuses à usage médicinal appartenant à 29 familles et à un grand groupe systématique à savoir les Magnoliophytes ont été recensées. Les familles les plus représentées dans les sept types utilisables de terres sont: Asteraceae (8 espèces), Apocynaceae (6 espèces), Euphorbiaceae (11 espèces), Fabaceae (5 espèces), Annonaceae (5 espèces), Caesalpiniaceae (4 espèces).

Tableau 1 : inventaire de grande famille de PFNL médicinal exploité

\begin{tabular}{|c|c|c|c|c|}
\hline Groupe systématique & Familles & Pourcentage (\%) & Espèces & Pourcentage (\%) \\
\hline Magnoliophytes & & & & \\
\hline Monocotylédones & 08 & 28 & 17 & 19 \\
\hline Dicotylédones & 21 & 72 & 74 & 81 \\
\hline Total & $\mathbf{2 9}$ & $\mathbf{1 0 0}$ & $\mathbf{9 1}$ & $\mathbf{1 0 0}$ \\
\hline
\end{tabular}

\section{Formes morphologiques des PFNL médicinaux}

Les espèces forestières non ligneuses à l'usage médicinal recensé se regroupent au sein de quatre (4) types morphologiques présentés ci-dessous. Les arbres $(58 \%)$ représentent le type morphologique le plus dominant, suivi des herbacées $(19 \%)$. Les types morphologiques «lianes » et les «arbustes » présentent respectivement $(15 \%)$ et $(08 \%)$. Ces différents types morphologiques offrent différents PFNL exploités à des degrés divers (intensif ou moins intensif) en fonction de la demande domestique et/ou de la valeur commerciale du produit par les populations enquêtées.

Tableau 2: inventaire des types morphologiques des PFNL recensés

\begin{tabular}{|l|c|c|}
\hline \multicolumn{1}{|c|}{ Types morphologiques } & Nombre d'espèces & Pourcentage (\%) \\
\hline Arbres & 53 & 58 \\
\hline Arbustes & 14 & 15 \\
\hline Herbacées & 17 & 19 \\
\hline Lianes & 7 & 8 \\
\hline Total & $\mathbf{9 1}$ & $\mathbf{1 0 0}$ \\
\hline
\end{tabular}

\section{Différents Types d'Utilisation des Terres (TUT) et la richesse spécifique des PFNL}

Différents facteurs pédoclimatiques, biologiques et anthropiques influencent l'évolution des écosystèmes forestiers. Chaque état d'évolution 
des systèmes impose une forme d'utilisation et de gestion de l'espace. Certains de ces formes ou types (TUT) se trouvent être des réponses à des situations résultant de perturbations de ces écosystèmes par les feux de brousse, des invasions d'insectes ou du développement de certaines maladies, sans bien sûr oublier les interventions intempestives des hommes (exploitation forestière, activités agricoles normales, etc.). D'autres TUT sont tout simplement un état d'évolution intermédiaire d'une formation végétale dans ses mouvements cycliques vers l'atteinte du climax.

Sept (07) TUT ont été identifiés dans le cadre de cette étude. Il s'agit : la forêt secondaire âgée, la forêt secondaire jeune, la jachère âgée, la jachère jeune, la plantation caféière et le marécage. Il ressort qu'à partir des données collectées que, la forêt secondaire jeune présente une richesse spécifique moyenne de 26,24\% constitue le TUT le plus riche en PFNL médicinal connues par la population que les autres TUT. D'autres TUT, la jachère jeune en l'occurrence, et la plantation caféière (une sorte d'agroforêt où plusieurs espèces à usage médicinal sont conservées), montrent une richesse spécifique proche de celle de la forêt secondaire jeune (soit $24,66 \%$ et $21,82 \%$ d'espèces respectivement). Puis suit la forêt secondaire âgée avec une richesse spécifique moyenne de $19,03 \%$ et la jachère âgée avec $10,06 \%$ de richesse ; enfin le marécage $3,80 \%$ se présentent comme le TUT le moins riche en plantes médicinales connues par la population. Ces TUT constituent des milieux écologiques fortement anthropisés. Cette anthropisation serait responsable de l'accroissement de la richesse spécifique de ces milieux ayant subi une recolonisation en espèces héliophiles à croissance rapide et très souvent à bois mou comme le cas de la jachère jeune (Musanga cecropiodes, Pycnathus angolensis, etc...). Or ces espèces à croissance rapide constituent des espèces pionnières pour l'établissement en sous-bois de différentes espèces forestières pérennes, mais à croissance lente.

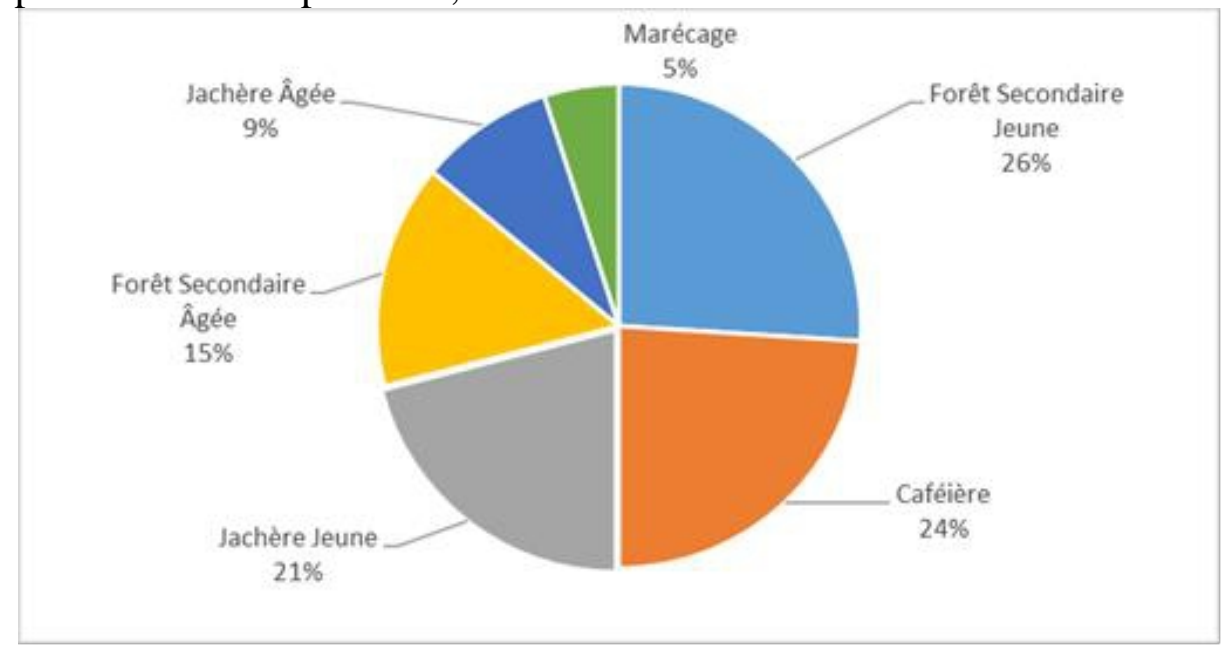

Figure $\mathbf{n}^{\circ} \mathbf{2}$ : répartition de la richesse spécifique par TUT 


\section{Utilité des PFNL médicinaux dans le traitement des maladies, symptômes et affections}

Les enquêtes réalisées dans les neuf (9) villages ont fait état de la prévalence de plus d'une vingtaine de maladies, symptômes et affections qui sont traitées à base des PFNL médicinaux au sein des populations concernées. Parmi les maladies les plus courantes, on a recensé (i) le paludisme (près de 95\% des cas) qui est une maladie grave biologiquement provoquée par un moustique du genre Anophèle. Le paludisme est à la cause de la moitié des cas de mortalité infantile dans le Sud-Ouest de la RCA (beaucoup d'enfants ne dorment pas sous des moustiquaires imprégnées) (ii) les amibiases et autres parasitoses intestinales (64\%), et (iii) le mal d'estomac (54\%); ces deux types d'affections provoquent des troubles digestifs et l'affaiblissement du corps; elles se développent à la faveur des effets de certains facteurs environnementaux et par un manque des mesures d'hygiènes appropriées. Il est important de noter le rhume $(61,4 \%)$, la toux $(54 \%)$ et la grippe $(54 \%)$ qui constituent des maladies et affections souvent endémiques en zone forestière. On note également les cas de maladies et affections qui ont une fréquence d'apparition comprise entre 20-30\%, il s'agit respectivement de la diarrhée, les maux de tête, l'anémie, la carie dentaire, la hernie, les blessures ou plaies, les MST et problèmes gynécologiques et la fièvre typhoïde. Enfin les maladies ayant une fréquence d'apparition inférieure à $20 \%$, il s'agit respectivement des symptômes de jaunisse, la rougeole, les abcès et le rhumatisme qui affecte le plus souvent les vieillards.

Pour la majorité de la population concernée, le seul espoir de guérison de toutes ces maladies repose sur les plantes médicinales non ligneuses.

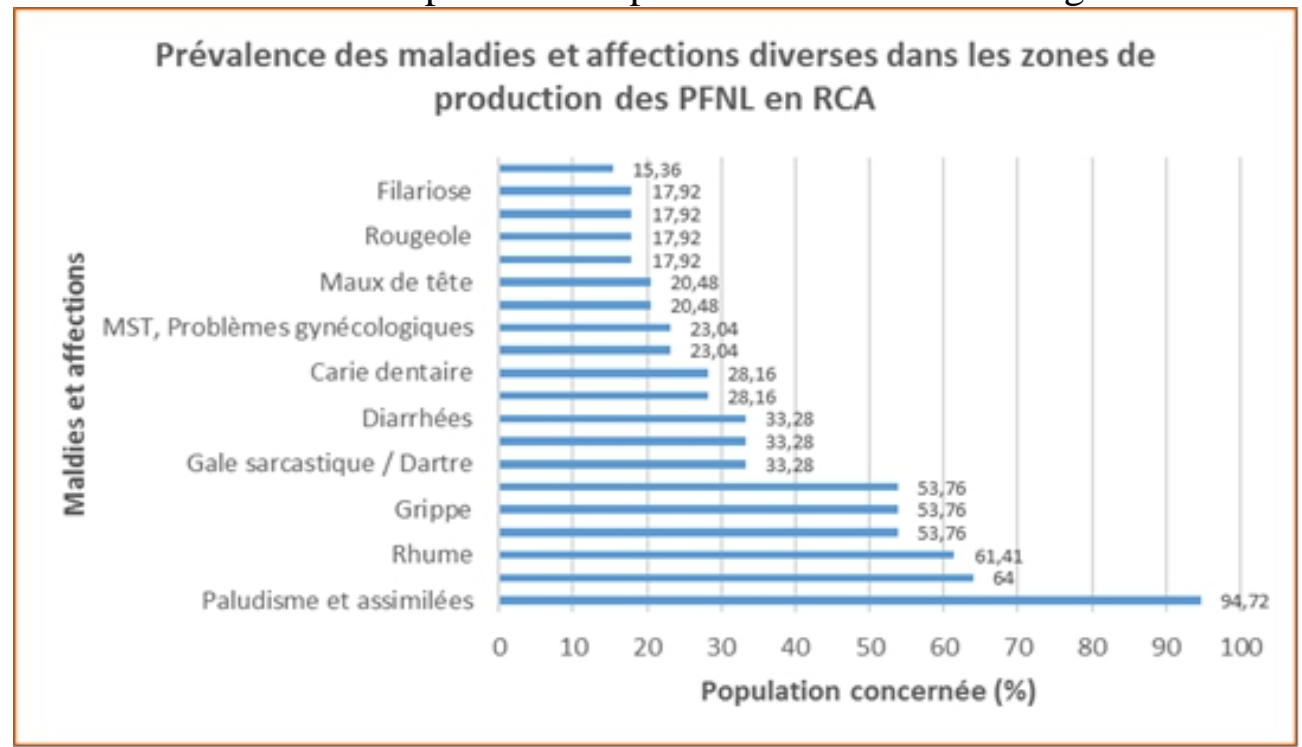

Figure 3 : maladies, symptômes et affections recensées dans les 9 villages enquêtés 


\section{Moyens de recours thérapeutiques}

Les données collectées montrent que $91,16 \%$ des personnes interrogées utilisent les plantes médicinales comme recours thérapeutique pour les soins de santé primaires lorsqu'elles sont affectées par la maladie. Ce recours aux plantes médicinales est dû à un taux de pauvreté élevé en milieu rural et à l'absence de structures sanitaires (case de santé) dans la zone d'étude.

Toutefois, il est important de signaler la présence d'un petit noyau $(<9 \%)$ de malades faisant recours aux pharmacies [soit de rue $(5,3 \%)$ avec les risques encourus, soit conventionnelles beaucoup plus rassurantes $(3,5 \%)$ ] pour leurs soins médicaux. Ces dernières années (2010-2012), certaines boutiques s'intéressent de plus en plus à la vente des médicaments pharmaceutiques, bien que de manières informelle et très souvent illicite. Ce phénomène a encouragé le développement de l'automédication dans la zone.

Expression des choix de soins de santé par la population des zones de production des PFNL

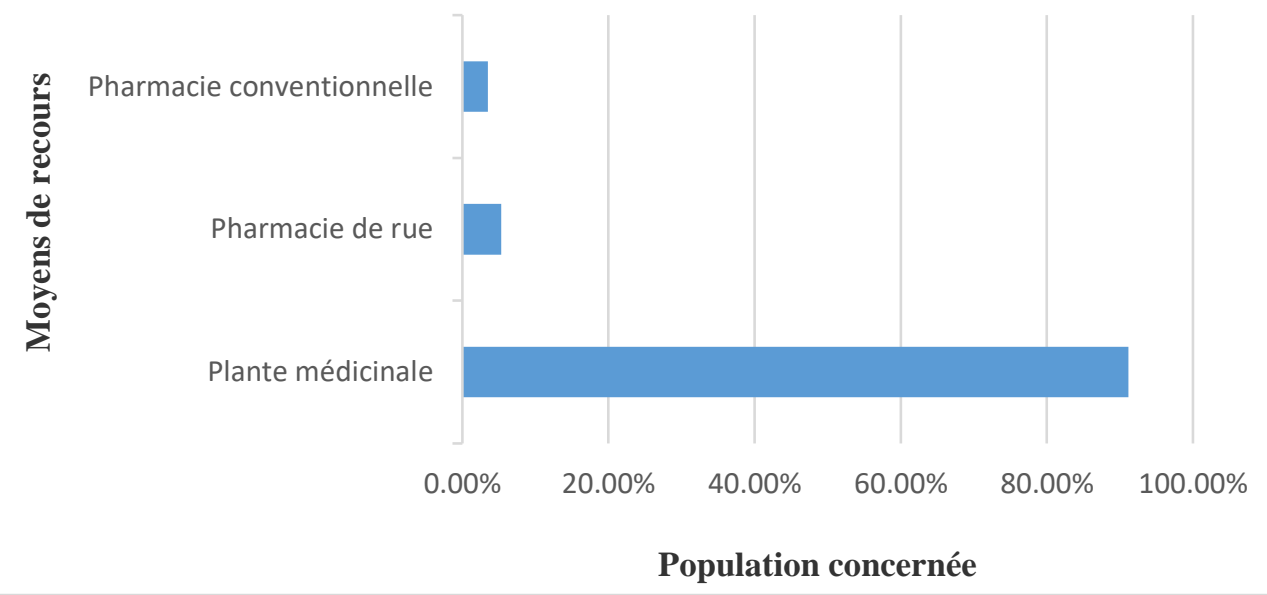

Figure 4 : moyens de recours thérapeutiques

\section{Parties des PFNL utilisés pour les soins médicaux et formes d'administration Parties des PFNL utilisés}

Les écorces, les feuilles, les fruits, les tiges, la sève, les racines et les bulbes constituaient les différentes parties des plantes sollicitées par la population de neuf villages enquêtés. Sur les 91 espèces végétales non ligneuses inventoriées, les écorces $(43,26 \%)$ et les feuilles $(39,21 \%)$ sont les organes les plus utilisés par les populations. Les fruits/graines $(5,07 \%)$ et les racines $(6,02 \%)$ sont utilisés comme médicament; mais les techniques de prélèvement et/ou de déracinement des racines influencent sur le processus physiologique des espèces. Les autres parties telles que les bulbes et les 
tubercules sont moins sollicitées par la pharmacopée (soit 0,16\% des individus pour les bulbes et $0,20 \%$ des individus pour les tubercules).

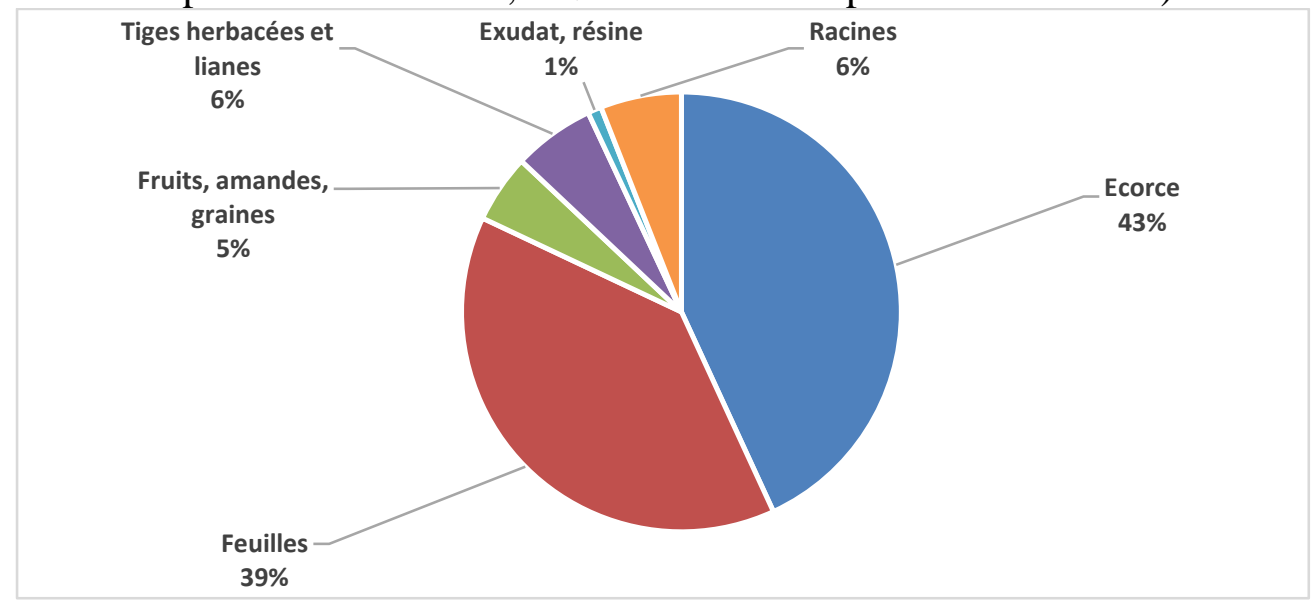

Figure 5 : différentes parties des PFNL utilisées en médecine traditionnelle

\section{Formes d'administration des PFNL médicinaux}

Les organes utilisés pour les traitements permettent d'obtenir généralement des produits finaux se présentant sous trois formes : liquides (macéré, décocté, infusé, etc.), poudre et pâte. Ces formes sont obtenues suivant différents modes opératoires appliqués sur différentes parties et organes en fonction de la pathologie. Cinq modes opératoires sont fréquemment pratiqués pour l'obtention des produits de traitement dans les 9 villages enquêtés : l'infusion, la décoction, la macération, l'expression et l'incinération/broyage.

\section{a. La macération}

Elle consiste à laisser en contact les organes des plantes avec un solvant pendant un temps assez long. Dans la plupart de cas, on utilise l'eau ou le vin pendant plus ou moins 24 heures. Les organes des plantes sont soit triturés ou coupés en morceaux pour favoriser l'extraction de la matière active. La solution ainsi obtenue est un macéré. C'est le cas de macéré de Rauvolfia vomitoria; Pycnanthus angolensis.

\section{b. La décoction}

Elle consiste à faire bouillir les organes végétaux entiers ou coupés, dans un solvant (eau ou vin de canne à sucre) pendant un temps bien déterminé (10 minutes à 1heure et demie). La solution ainsi obtenue est un décocté. La décoction a fait aussi l'objet de l'apprentissage par essais et par erreurs, par la plupart de nos enquêteurs surtout pour combattre la fièvre, la toux et l'amibiase. Par exemple, l'utilisation de l'Euphorbia hirta en décoction contre les vers intestinaux et les amibes. Il en est de même des espèces Lantana 
camara et Jatropha curcas L. contre la toux, la fièvre et la desquamation de la gorge et de l'espèce Cassia occidentalis contre la malaria.

\section{c. L'Infusion}

Elle consiste à mettre en contact pendant quelques minutes une plante ou les organes des plantes avec de l'eau bouillante ou chaude. Le produit ainsi obtenu est l'infusé. L'exemple le plus concret est l'usage des feuilles de l'espèce Lippia multiflora en infusion pour soigner la toux. Cette pratique a été observée dans la plupart des villages enquêtés.

\section{d. L'expression}

C'est un procédé qui consiste en l'extraction du suc des végétaux frais soit par écrasement au mortier des organes végétaux, soit en pressant. Le suc ainsi obtenu est utilisé pour les instillations nasales, oculaires ou auriculaires. Le cas le plus fréquent est l'usage des feuilles de l'espèce Kalanchoe pinnata et Jatropha curcas pour soigner l'otite dans les zones enquêtées.

\section{e. L'incinération}

C'est la décomposition des organes végétaux par la chaleur, qui sont ensuite broyés ou pilés. Généralement, le matériel végétal est préalablement raclé ou séché au soleil. Ce mode de préparation a été observé dans le cadre de la fabrication du sel indigène à base d'Hydrocharis chevalieri, pour le bicarbonate indigène «l'alkalis» à base des inflorescences des palmiers, et aussi pour des pulpes des plantains pour soigner diverses formes d'hémorroïdes et certaines maladies respiratoires.

Les produits obtenus à partir de ces différents modes de préparation sont administrés de diverses manières :

- Pour les usages internes : par les voies orale, rectale, vaginale ou urétrale et aussi à travers le gargarisme (rinçage sans avaler le liquide) ;

- Pour les usages externes : par la friction, le bain corporel, le bain de vapeurs, les voies nasale, auriculaire et oculaire.

\section{Les plantes médicinales prioritaires dans le Sud-Ouest de la RCA}

La priorisation a été faite à base de la méthode des scores. Le score représente la valeur d'usage ethnobotanique pour évaluer l'importance de l'espèce au niveau médicinal. Les variables recueillies selon les types d'usages répertoriés des espèces, permettent de les classer selon un score allant de 1 à 5 points ( 5 pour l'espèce la plus appréciée; 4 pour la suivante et ainsi de suite...).

Suite aux résultats obtenus sur le terrain, des fiches de priorisation des PFNL médicinaux ont été établies. Il est important de souligner qu'un PFNL 
est dit prioritaire si, sur le plan alimentaire, médical, commercial ou autre, il présente différents intérêts non négligeables pour une population donnée. Les fiches de priorisation ont été élaborées en tenant compte des critères socioéconomiques, environnementaux et culturels que revêt chaque PFNL. Le tableau ci-dessous présente les 19 PFNL médicinaux prioritaires recensés.

Tableau 3 : Liste des PFNL médicinaux prioritaire dans le Sud-Ouest de la RCA

\begin{tabular}{|c|c|c|c|c|c|c|}
\hline $\mathbf{N}^{\circ}$ & Nom scientifique & Famille & $\begin{array}{l}\text { Parties } \\
\text { prélevées }\end{array}$ & $\begin{array}{l}\text { Mode de } \\
\text { préparation }\end{array}$ & $\begin{array}{l}\text { Importance } \\
\text { thérapeutique }\end{array}$ & $\begin{array}{l}\text { Mode } \\
\text { d'exploitation }\end{array}$ \\
\hline 01 & Jatropha curcas & Euphorbiaceae & $\begin{array}{l}\text { Feuilles, } \\
\text { grains, } \\
\text { racines }\end{array}$ & $\begin{array}{l}\text { Décoction, } \\
\text { expression }\end{array}$ & $\begin{array}{l}\text { Toux, Dermatose, } \\
\text { Paludisme }\end{array}$ & $\begin{array}{l}\text { Cueillette, } \\
\text { ramassage }\end{array}$ \\
\hline 02 & $\begin{array}{l}\text { Chromolaena } \\
\text { odorata }\end{array}$ & Asteraceae & $\begin{array}{l}\text { Feuilles } \\
\text { Racines }\end{array}$ & $\begin{array}{l}\text { Décoction, } \\
\text { expression }\end{array}$ & $\begin{array}{l}\text { mal de } \\
\text { conjonctivite, } \\
\text { typhoïde }\end{array}$ & Cueillette \\
\hline 03 & Euphorbia hirta & Euphorbiaceae & Feuilles & Décoction & Amibiases, diarrhées & Cueillette \\
\hline 04 & $\begin{array}{l}\text { Ranvolfia } \\
\text { vomitoria }\end{array}$ & Apocynaceae & $\begin{array}{l}\text { Ecorce, } \\
\text { Feuilles, } \\
\text { Racine }\end{array}$ & $\begin{array}{l}\text { Décoction, } \\
\text { infusion }\end{array}$ & $\begin{array}{l}\text { Paludisme, maux de } \\
\text { dent, }\end{array}$ & $\begin{array}{l}\text { Cueillette, } \\
\text { Ecorçage }\end{array}$ \\
\hline 05 & Alstonia boonei & Apocynaceae & Ecorces & Decoction, & Fièvre, paludisme, & Ecorçage \\
\hline 06 & Lophira alata & Ochnaceae & Feuilles & Decoction & $\begin{array}{ll}\text { paludisme, } & \text { carie } \\
\text { dentaire } & \end{array}$ & Cueillette \\
\hline 07 & $\begin{array}{l}\text { Musanga } \\
\text { cecropioides }\end{array}$ & Cecropiaceae & $\begin{array}{l}\text { Feuilles, } \\
\text { écorces }\end{array}$ & Décoction & $\begin{array}{l}\text { abcès, rhume, toux, } \\
\text { mal d'estomac, grippe. }\end{array}$ & $\begin{array}{l}\text { Cueillette, } \\
\text { Ecorçage }\end{array}$ \\
\hline 08 & $\begin{array}{l}\text { Monodora } \\
\text { myristica }\end{array}$ & Annonaceae & $\begin{array}{l}\text { Fruits, } \\
\text { feuilles }\end{array}$ & Consommé cru, & $\begin{array}{l}\text { anémie, hémorroïdes, } \\
\text { faiblesse sexuelle, }\end{array}$ & Cueillette \\
\hline 09 & Piper guineenses & Piperaceae & Feuille, tige & Décoction & $\begin{array}{l}\text { Anémie, } \\
\text { sexuelle }\end{array} \quad$ faiblesse & Cueillette \\
\hline 10 & $\begin{array}{l}\text { Annona } \\
\text { senegalensis }\end{array}$ & Annonaceae & Feuilles & $\begin{array}{l}\text { Décoction, } \\
\text { macération }\end{array}$ & Paludisme, rougeole & Cueillette \\
\hline 11 & Morinda lucida & Rubiaceae & $\begin{array}{l}\text { Feuilles, } \\
\text { Ecorces, }\end{array}$ & Décoction, & $\begin{array}{l}\text { Paludisme, Typhoïde, } \\
\text { faiblesse sexuelle }\end{array}$ & Cueillette \\
\hline 12 & Picralima nitida & Apocynaceae & Ecorces & Décoction & $\begin{array}{ll}\begin{array}{l}\text { Paludisme, } \\
\text { typhoïde }\end{array} & \text { fièvre } \\
\end{array}$ & Ecorçage \\
\hline 13 & Annikia chlorantha & Annonaceae & Ecorces & Décoction & $\begin{array}{l}\text { Fièvre jaune, } \\
\text { paludisme, hépatite B, }\end{array}$ & Ecorçage \\
\hline 14 & Aloe vera & Moringaceae & Suc, feuilles & $\begin{array}{l}\text { Décoction, } \\
\text { expression }\end{array}$ & $\begin{array}{l}\text { Abcès, plaies, } \\
\text { paludisme, dermatose, }\end{array}$ & Cueillette \\
\hline 15 & Cola nitida & Sterculiaceae & $\begin{array}{l}\text { Feuilles, } \\
\text { Fruits, } \\
\text { Ecorces, }\end{array}$ & $\begin{array}{l}\text { Décoction, } \\
\text { consommée } \\
\text { crue }\end{array}$ & $\begin{array}{l}\text { Mal de tête, propriétés } \\
\text { aphrodisiaques, }\end{array}$ & $\begin{array}{l}\text { Cueillette, } \\
\text { ramassage, }\end{array}$ \\
\hline 16 & $\begin{array}{l}\text { Vermonia } \\
\text { amygdalina }\end{array}$ & Asteraceae & Feuilles & Macération & $\begin{array}{l}\text { Mal d'estomac, } \\
\text { dermatose }\end{array}$ & Cueillette \\
\hline 17 & $\begin{array}{l}\text { Myrianthus } \\
\text { arborus }\end{array}$ & Moraceae & $\begin{array}{l}\text { Fruits, } \\
\text { feuilles }\end{array}$ & $\begin{array}{l}\text { Décoction, } \\
\text { consommée } \\
\text { crue }\end{array}$ & $\begin{array}{l}\text { Maux de ventre, } \\
\text { anémie, maux de } \\
\text { dents, }\end{array}$ & Cueillette \\
\hline 18 & $\begin{array}{l}\text { Tabernaemontana } \\
\text { crassa }\end{array}$ & Apocynaceae & Sève, écorces & $\begin{array}{l}\text { Décoction, } \\
\text { expression }\end{array}$ & $\begin{array}{l}\text { Paludisme, abcès, } \\
\text { blessures, anémie. }\end{array}$ & Cueillette \\
\hline 19 & $\begin{array}{l}\text { Penianthus } \\
\text { longifolius }\end{array}$ & Menispermaceae & $\begin{array}{l}\text { Ecorces, } \\
\text { racines }\end{array}$ & $\begin{array}{l}\text { Décoction, } \\
\text { consommée } \\
\text { crue }\end{array}$ & $\begin{array}{l}\text { Paludisme, propriétés } \\
\text { aphrodisiaques }\end{array}$ & cueillette \\
\hline
\end{tabular}




\section{Impacts des activités humaines sur les PFNL médicinaux prioritaires}

Les impacts des activités humaines sur la niche écologique des espèces forestières non ligneuses sont néfastes et conduisent à une érosion génétique de ces ressources à fort potentiel socio-économique. Les données obtenues et analysées ont permis de relever la disparition progressive de certaines espèces forestières non ligneuses à usage médical tel que : Monodora myristica, Piper guineenses, Morinda lucida, Picralima nitida, Annikia chlorantha, Khaya anthoteca. et Cola nitida avec une valeur comprise entre 83,63 - 97,26\%. Cette disparition est due par une utilisation intense de ces espèces par les populations habitant les neuf villages et les intérêts que présentent ces espèces dans le cadre de la pharmacopée traditionnelle.

Tableau 4: Liste des PFNL médicinaux en voie d'extinction

\begin{tabular}{|l|l|l|l|l|l|}
\hline $\mathbf{N}^{\circ}$ & Nom scientifique & Famille & Pourcentage & Priorité & Problèmes identifiés \\
\hline 01 & Picralima nitida & Annonaceae & 97,26 & 5 & Exploitation abusive \\
\hline 02 & Alstonia boonei & Apocynaceae & 96,57 & 5 & Exploitation abuse \\
\hline 03 & Annikia chlorantha & Rubiaceae & 96.26 & 5 & Exploitation abuse \\
\hline 04 & Piper guineenses & Apocynaceae & 94.13 & 4 & Exploitation abuse \\
\hline 05 & Monodora myristica & Annonaceae & 91.62 & 5 & Exploitation abuse \\
\hline O6 & Penianthus longifolius & Menispermaceae & 90.86 & 4 & Exploitation abuse \\
\hline 07 & Cola nitida & Sterculaceae & 83,63 & 4 & Exploitation abuse \\
\hline
\end{tabular}

$N . B: 5=1^{\text {ère }}$ priorité $; 4=2^{\text {ème }}$ priorité $; 3=3^{\text {ème }}$ priorité..

Il résulte d'une manière générale, que la double sollicitation de Cola $s p$. en alimentation et en médecine entraînera en cout terme son érosion dans la zone sud-ouest de la RCA par rapport aux autres PFNL. Des stratégies de conservation doivent être prises pour garantir la durabilité de cette espèce au profit de la génération future, car sa demande est quotidienne.

\section{Impacts des activités humaines sur le mode d'exploitation des PFNL médicinaux}

L'exploitation des PFNL à usage médicinal dans le sud-ouest de la RCA est fonction de la demande domestique ou commerciale du produit concerné. L'impact de cette exploitation sur la structure et la composition de la niche écologique est étroitement lié au degré d'utilisation de l'espèce, mais aussi à l'organe végétal prélevé (fruits, feuilles, écorces, racines, exsudats, tiges...). Dans le cadre de cette étude, les paramètres d'évaluation d'impact utilisés M. Tchatat et O.Ndoye (2006) ont été adoptés pour apprécier (i) la quantité du produit à exploiter, (ii) la technique de prélèvement de l'organe récolté, et (ii) la fréquence de prélèvement. 


\section{a. Les Feuilles}

Plusieurs méthodes ont été utilisées par les ménages enquêtés pour s'acquérir des feuilles des espèces fournissant des PFNL médicinaux. Les impacts relevés sur la ressource varient souvent selon les espèces.

Tableau 5 : Impact de l'exploitation des feuilles sur la ressource en fonction du type biologique

\begin{tabular}{|l|l|l|l|l|}
\hline $\begin{array}{l}\text { Types } \\
\text { biologiques }\end{array}$ & Méthode de récolte & Période & $\begin{array}{l}\text { Impact sur la } \\
\text { ressource }\end{array}$ & Espèces identifiées \\
\hline $\begin{array}{l}\text { Arbuste/petit } \\
\text { arbre/jeune arbre }\end{array}$ & Arbre sur pied & Permanent & ++++ & $\begin{array}{l}\text { Dostenia psilumus } \\
\text { Myrianthus arboreus } \\
\text { Cola nitida }\end{array}$ \\
\hline Herbacées & Arbre sur pied & Permanent & +++++ & $\begin{array}{l}\text { Megaphrynium macrostachyum } \\
\text { Piper umbellatum }\end{array}$ \\
\hline Lianes & Arbre sur pied & Permanent & +++ & Piper guineensis \\
\hline
\end{tabular}

$$
+: \text { très faible } ;++ \text { : faible } ;+++: \text { fort } ;++++: \text { très fort. }
$$

\section{b. Les fruits}

La méthode utilisée pour récolter les fruits est fonction de la taille de l'arbre ou de l'arbuste. L'exploitation fruitière des arbustes ou des jeunes arbres productifs destinée à l'autoconsommation provoque peu de dégâts qu'à usage sanitaire au sein de la structure forestière. Les cimes de ces arbres étant facilement accessibles, les fruits sont récoltés soit en grimpant sur l'arbre, soit à l'aide d'une perche. Lorsque l'arbre est devenu trop grand et qu'il n'est plus accessible aux cueilleurs, deux options sont utilisées par les populations des zones enquêtées :

$\checkmark$ attendre la chute des fruits (41\% des ménages enquêtés);

$\checkmark$ abattre l'arbre afin de les récolter facilement (59\% des ménages surtout les commerçants)

Cette dernière méthode présente un impact néfaste sur le cycle biologique de l'arbre et malheureusement adopté pour les arbres producteurs dont les fruits dépérissent rapidement, avec une maturation physiologique (et commerciale) simultanée et massive. C'est le cas d'Annonidium mannii. Dans la zone d'étude, on a constaté aussi que le Penianthus longifolius, qui est un petit arbuste de sous-bois dont l'écorce et surtout la racine sont très prisées par les populations pour ses effets aphrodisiaques, est exploité selon cette méthode. Sa récolte malheureusement consiste à arracher l'arbuste ou à couper la partie souterraine, car selon les récolteurs c'est dans cette partie où se concentrerait la «molécule active ». Si ce rythme de récolte se poursuit, la disparition de l'espèce est garantie pour les prochaines années. C'est pourquoi une bonne compréhension des stratégies de reproduction, de la structure, de la densité et de la distribution de la population de cette espèce est nécessaire pour une bonne gestion. 
Tableau 6 : Impact de l'exploitation des fruits sur la ressource en fonction du type biologique

\begin{tabular}{|l|l|l|l|l|l|}
\hline $\begin{array}{l}\text { Parties } \\
\text { utilisées }\end{array}$ & $\begin{array}{l}\text { Types } \\
\text { biologiques }\end{array}$ & $\begin{array}{l}\text { Méthode de } \\
\text { récolte }\end{array}$ & Période & $\begin{array}{l}\text { Impact sur la } \\
\text { ressource }\end{array}$ & $\begin{array}{l}\text { Espèces } \\
\text { identifiées }\end{array}$ \\
\hline $\begin{array}{l}\text { Pulpe/aman } \\
\text { de }\end{array}$ & $\begin{array}{l}\text { Arbuste/petit } \\
\text { arbre/jeune arbre }\end{array}$ & $\begin{array}{l}\text { Arbre sur pied ou } \\
\text { arrachage du } \\
\text { pied }\end{array}$ & Saisonnière & +++++ & $\begin{array}{l}\text { Garcina lucida; } \\
\text { Coula edulis } \\
\text { Penianthus } \\
\text { longifolius }\end{array}$ \\
\hline Amande & $\begin{array}{l}\text { Arbre/grand } \\
\text { arbre }\end{array}$ & $\begin{array}{l}\text { Ramassage } \\
\text { partiel ou total } \\
\text { des fruits }\end{array}$ & Saisonnière & +++ & $\begin{array}{l}\text { Ricinodendron } \\
\text { heudelotii, } \\
\text { Irvingia } \\
\text { gabonensis }\end{array}$ \\
\hline Pulpe & $\begin{array}{l}\text { Arbre/grand } \\
\text { arbre }\end{array}$ & $\begin{array}{l}\text { Arbre sur pied ou } \\
\text { après abattage }\end{array}$ & Saisonnière & +++++ & $\begin{array}{l}\text { Annonidium } \\
\text { mannii }\end{array}$ \\
\hline
\end{tabular}

$+:$ très faible $;++:$ faible $;+++:$ fort $;+++++$ : très fort.

\section{c. Les écorces, racines, tubercules et tiges}

Ces organes sont exploités de différentes manières en fonction des espèces. Les procédures utilisées pouvant avoir des répercussions néfastes sur la plante et son écosystème.

Tableau 7 : Impact de l'exploitation des écorces, des racines et des tiges sur la ressource en fonction du type biologique

\begin{tabular}{|c|c|c|c|c|c|}
\hline $\begin{array}{l}\text { Parties } \\
\text { utilisées }\end{array}$ & $\begin{array}{l}\text { Types } \\
\text { biologiques }\end{array}$ & Méthode de récolte & Période & $\begin{array}{l}\text { Impact sur } \\
\text { la } \\
\text { ressource }\end{array}$ & $\begin{array}{l}\text { Espèces } \\
\text { identifiées }\end{array}$ \\
\hline Écorce & $\begin{array}{l}\text { Arbuste/petit } \\
\text { arbre/arbre/grand } \\
\text { arbre }\end{array}$ & $\begin{array}{l}\text { Arbre sur pied ou } \\
\text { après abattage }\end{array}$ & Permanent & +++++ & Garcina lucida; \\
\hline Racine & $\begin{array}{l}\text { Arbuste/petit } \\
\text { arbre }\end{array}$ & $\begin{array}{l}\text { Après abattage de } \\
\text { l'arbre }\end{array}$ & Permanent & +++++ & $\begin{array}{l}\text { Penianthus } \\
\text { longifolius } \\
\text { Garcina lucida }\end{array}$ \\
\hline Tubercule & Liane & Déracinement partiel & Permanent & +++++ & $\begin{array}{l}\text { Dioscorea } \\
\text { liebrechtsiana }\end{array}$ \\
\hline Tige & $\begin{array}{l}\text { Arbuste/petit } \\
\text { arbre/arbre }\end{array}$ & Abattage & Permanent & +++++ & $\begin{array}{l}\text { Xylopia } \\
\text { aethiopica, } \\
\text { Paulinia pinnata }\end{array}$ \\
\hline Sève & $\begin{array}{l}\text { Arbre sur pied } \\
\text { ou abattage }\end{array}$ & $\begin{array}{l}\text { Arbre sur pied ou } \\
\text { abattage }\end{array}$ & Permanent & +++++ & $\begin{array}{l}\text { Raphia ssp } \\
\text { Elais guinenses }\end{array}$ \\
\hline
\end{tabular}

$+:$ très faible $;++$ : faible $;+++:$ fort $;++++$ : très fort.

Le prélèvement des écorces et des tiges laisse des impacts élevés et observables sur le terrain. En fonction du type biologique, le prélèvement des racines est parfois élevé, tandis qu'il est peu important pour les tubercules.

Il est important de relever que l'écorçage devrait faire l'objet d'une sensibilisation auprès des populations, car le cernage autour du tronc d'arbre est une pratique d'exploitation non durable de la ressource. 


\section{Discussion}

La forêt du Sud-Ouest de la RCA est un habitat diversifié en PFNL à usage médicinal recherché par les populations riveraines. Cette étude a permis de dégager l'importance que revêtent les espèces végétales non ligneuses dans les soins sanitaires des populations vivant dans ou à la périphérie des neuf (9) villages enquêtés et les stratégies mises en place pour atténuer la dégradation de ces espèces dues aux impacts des activités anthropiques sur leur durabilité. Les résultats obtenus montrent que les connaissances liées à l'utilisation de ces PFNL inventoriés dans les sept (7) types d'utilisations de terre (TUT) varient suivant les groupes socioculturels. Cette différence de connaissance pourrait s'expliquer par les origines diverses de chacun de ces groupes ethniques. Ceci démontre que l'utilisation des PFNL à usage médicinal est parfois fonction des facteurs culturels et confirme le constat Akogo (2002) ; car, bien qu'étant dans le même milieu, les populations connaissent et utilisent différemment les ressources du milieu.

L'inventaire réalisé dans les sept TUT a permis de recenser 91 espèces végétales non ligneuses à usage médicinal appartenant à 29 familles et à un grand groupe systématique à savoir les Magnoliophytes. L'usage relatif aux organes végétaux par les populations riveraines révèle que les écorces (43\%), suivie des feuilles $(39 \%)$ et les racines $(6 \%)$ représentent la partie la plus utilisée par la population pour soigner des maladies, des affections et symptômes. La macération, l'infusion, la décoction et l'expression sont les principales méthodes utilisées par les patients pour extraire le principe actif des organes prélevés. Ces données sont avoisinantes à celles obtenues par Essomba (2009), dans son étude sur «Approche participative pour une utilisation durable de la flore médicinale en zone forestière du Sud-Cameroun cas du terroir de Tya'assono (vallée du Ntem), auxquelles 98 espèces végétales non ligneuses ont été recensées. Les écorces $(51,91 \%)$, les feuilles $(33,46 \%)$ et les racines $(7 \%)$ constituent la partie utilisée par les populations du sudCameroun pour se soigner, ainsi qu'au Gabon par Ngoye (2010) et Anonyme (2006).

Ces différents usages identifiés reposent sur le niveau de connaissance des enquêtes concernant l'exploitation des PFNL médicinaux ayant permis d'identifier 19 espèces végétales non ligneuses prioritaires dont 07 de ces espèces sont en voie de dégradation. On lie cette rapide disparition aux activités humaines incontrôlées. Elles représentent une sérieuse menace pour l'existence de la biodiversité végétale. L'importance accordée à une espèce ne dépend pas de sa disponibilité, mais de sa capacité à satisfaire les besoins socioéconomiques des populations (Bonané; 2002). N'gassé (2010) mentionne dans la revue nationale la disparition des PFNL dans le sud-ouest de la RCA. 
Les populations de cette région font preuve de passivité et exercent une forte pression sur ces ressources naturelles. Les prélèvements des racines et des écorces semblent avoir plus d'incidences écologiques néfastes que les fruits et les feuilles. Cependant, la récolte des feuilles et des fruits pourrait aussi avoir un impact sur le processus de régénération naturelle et la conservation de l'espèce, car, selon Mialoundama, 2002, le maintien de la capacité de régénération dépend entre autres du maintien des principaux principes de la régénération tels que la pollinisation, le développement et la dispersion des semences, la germination et la croissance des plantes. Toujours selon ce même auteur, les semences de certaines espèces sont récalcitrantes et perdent très rapidement leur pouvoir germinatif après la récolte. Aussi, compte tenu de l'importance socio-économique et culturelle du Cola sp. les populations ont tendance à récolter l'ensemble de la production fruitière. Cette pratique empêche ou limite la régénération naturelle de l'espèce. Toutefois, ce n'est pas uniquement la partie prélevée qui importe, mais surtout comment la récolte est faite. Les modes d'exploitation les plus utilisés dans le milieu d'étude sont le prélèvement sur pied et le ramassage. Par contre, le ramassage est dangereux pour la dynamique de l'espèce si l'ensemble des produits est récolté.

Par ailleurs, certains usagers arrachent par ignorance, l'écorce tout autour de l'arbre surtout les espèces utilisées pour soigner le palu Alstonia boonei, Picralinia nitida, Annikia clorantha, Voacanga thouarsii, Tabernaemontana crassa et Pentaclethra macrophylla. Ce système de prélèvement pourrait entraîner de graves traumatismes à l'arbre allant jusqu'à la mort de celui-ci ; puisque l'écorce est la partie par laquelle l'arbre se nourrit. D'autre part, la récolte des feuilles et des écorces aura un impact beaucoup plus important si la plante est jeune que si elle est âgée. Les racines de Carica papaya, Chromolena odorata, Jatropha curcas, Penianthus longifolius, Carpolobia lutea sont utilisés par de nombreuses populations pour soigner la fièvre typhoïde, impuissance sexuelle, asthme, carie dentaire, vers intestinaux, MST (syphilis, gonococcie), diabète et hernie. Dans quatre régions du Congo Brazzaville, le décocté des racines de Carica papaya est utilisé en association avec les fruits de Citrus aurantifolia pour le traitement de la syphilis (Diafouka, 1997). Au Gabon, les racines de Penianthus longifolius sont prisées par les populations pour ses effets aphrodisiaques (Ngoye, 2010). Zima (2009) dans son étude sur «Intérêt socioéconomique de Jatropha curcas : Utilisation actuelle et potentielle dans les villes de Bangui et de Brazzaville » a montré que, le décocté aqueux des racines de Jatropha curcas est utilisé par les populations desdites villes pour soigner la carie dentaire, les parasites intestinaux, la fièvre typhoïde et la hernie. Cependant, la méthode de collecte de ces espèces n'est pas durable ; elle consiste à déraciner la plante entière afin de couper la partie souterraine contenant des molécules actives, cette méthode 
conduit après la collecte des organes à la mort et/ou à la disparition progressive de l'espèce. L'ouverture de la forêt du sud-ouest à l'exploitation forestière accélère entre autres la disparition et la raréfaction de plusieurs espèces végétales non ligneuses.

\section{Conclusion}

L'écosystème forestier du Sud-Ouest de la RCA possède une flore médicinale riche et contribue au moyen d'existence des populations rurales, généralement pauvres, qui vivent à l'intérieur ou à sa périphérie. Ses ressources biologiques variées sont utilisées de façon non durable par les populations locales. Les résultats obtenus dans le cadre de cette étude montrent que les PFNL à usage médicinal jouent un rôle très important dans le processus sanitaire des populations vivant dans le Sud-Ouest de la RCA. Les techniques de collecte des différentes parties de ces ressources utilisées par les populations conduisent à une dégradation de niche écologique de ces espèces. La définition d'une stratégie d'exploitation rationnelle des PFNL basée sur (i) la mise en application d'un plan national de développement des produits forestiers non ligneux qui est un outil subséquent révélant l'importance de ces derniers sur le plan économique, social, culturel et environnemental pour les populations locales, les Etats de l'espace COMIFAC et de la communauté internationale, (ii) la mise en application des textes réglementaires par les autorités centrafricaines concernant la gestion durable des ressources forestières non ligneuses, et (iii) les renforcements des capacités des acteurs sur les techniques de prélèvement écologique et socioéconomique des PFNL constituent des solutions idoines pour assurer une gestion durable de ces produits au profit des générations future.

\section{References:}

1. Abayomi S., (1996). Plantes médicinales et médecine traditionnelle d'Afrique. KARTHALA, Paris. 370 p.

2. Akogo G., (2002). Conservation et gestion durable des écosystèmes des forêts tropicales humides de l'Afrique Centrale : étude de cas d'aménagement forestier exemple en Afrique Centrale. La zone de Campo-Ma'an.FAO, Rome. pp. 19-20.

3. Anonyme., (2006). Assorti des plans de gestion des plantes médicinales et autres PFNL. PNDP, Yaoundé.105 p.

4. Bahuchet S, (1992). Dans la forêt d'Afrique Centrale les Pygmées Aka et Baka.Selaf, Paris, 640p

5. Bonanée M. (2002) : Gnetum buchholzianum et Piper Guinense en forêt de Ngotto. Le Flamboyant, $N^{\circ}$ 55, 4344.

6. Borrini-Feyerabend G., Farwar T., Nguinguiri C. et Ndangang V., (2000). La gestion participative des ressources naturelles, Organisation 
Négociation et Apprentissage par l'Action. GTZ et UICN, Heidelberg. $96 \mathrm{p}$.

7. BRENAN J., (1963). Utilité des flores pour les pays sous-développésimpact $3: 123-148$.

8. Diafouka P., (1997). Analyse des usages des plantes médicinales dans les quatre régions du Congo-Brazzaville. Thèse de Doctorat ès sciences. Université libre de Bruxelles. pp. 1-40.

9. Drabo-Ibrahima, (2006). Programme de Gestion des Ressources Forestières - MEVC. 86p.

10. Essomba D J P., (2009). Approche participative pour une utilisation durable de la flore médicinale en zone forestière du Sud-Cameroun : cas du terroir de Tya'assono (vallée du Ntem), 36pp.

11. FAO, (2003). Etude prospective du secteur forestier en Afrique: Rapport sous régional Afrique Centrale. Rome, 11pp.

12. Gounot M., (1969). Méthodes d'études quantitatives de la végétation. Masson, Paris. pp. 30-119.

13. Mialoundama F., (2002). Influence des feuilles sur la multiplication végétative par bouturage chez Gnetum africanum. Welw. Ann. Univ. Libre du Congo série B 1, 154-163.

14. N'gassé G., (2010). Revue Nationale sur les Produits Forestiers non Ligneux : PFNL (cas de la république centrafricaine : RCA), 63p.

15. Ngoye, A., (2010). Revue bibliographique sur les produits forestiers non ligneux (PFNL) : cas du Gabon, 59p PROTA, 2008

16. Toirambe B., (2005). Place des PFNL dans l'aménagement durable de la Réserve de Biosphère de Luki en R.DC. Travail de fin d'études. Gembloux : FUSAGx. 77p.

17. Zima G.G.S., (2009). Intérêt socio-économique du pourghère (Jatropha curcas. L) : Utilisations actuelles et potentielles dans les villes de Brazzaville (République du Congo) et de Bangui (République Centrafricaine). Mémoire de DEA, 59p. 